Gazi University
Journal of Science
http://dergipark.gov.tr/gujs

\title{
Effect of Inhomogeneity Constant on Equivalent Stresses in Elastic Analysis of Hollow Cylinder Made from Functionally Graded Material
}

\author{
Cem BOGA* \\ Adana Alparslan Türkeş Science and Technology University, Faculty of Engineering, Mechanical Engineering, 01250, Adana, Turkey
}

Highlights

- Elastic analysis of a cylinder made of functional grade material (FGM) was performed.

- Closed form analytical expressions are given radial displacement, radial and tangential stress values.

- Analytical results were found to be appropriate with Finite Element Method results.

- The obtained results, helps to prevent failure in the design of FGM hollow cylinder.

- The present results might be useful in designing FGM hollow cylinders.

\begin{tabular}{l} 
Article Info \\
\hline Received: $21 / 06 / 2019$ \\
Accepted: $17 / 09 / 2019$ \\
Keywords \\
\hline Equivalent stress \\
Functional graded material \\
Hollow cylinder \\
Inhomogeneity constant \\
Elastic analysis
\end{tabular}

\section{INTRODUCTION}

Structures like thick-walled shells, ship and submarine bodies, pressure vessels, portable pressure tubes, pressurized fluid passing pipes, autoclaves, booster systems, steam and central heating boilers are commonly used structural elements in engineering applications. Cylindrical pressured vessels have been the subject of many researchers in the past. The design of such structures is essential in order to fulfill their duties without being damaged. The stress distribution of the isotropic hollow circular cylinder exposed to inner and outer pressure is readily available in the literature from the formulas presented by Timoshenko and Goodier [1]. However, developing engineering applications have revealed the need for new materials and this has attracted the attention of many researchers. Especially in applications requiring high thermal resistance, high strength and thermal shock resistance, FGMs, which have all these properties together, have been observed to give better results compared to homogeneous materials. For designing of a FGM cylinder, a number of mathematical formulas were needed to be developed. And in the literature, there are many studies on elastic analysis of such FGM cylinders. Tütüncü and Temel [2] found radial deformations, radial and hoop stresses in FGM spheres, cylinders and disks exposed to uniform inner pressure for various material models using complementary functions method and plane elasticity equations. Boğa [3] presented the radial, tangential (hoop) stresses and radial displacements analytically and numerically (using ANSYS software) for four different types of FGM in the hollow a FGM cylinder subjected to internal pressure effect in his study. Li and Peng [4] analyzed FGM hollow disk and cylinder exposed to uniform internal pressure. Boğa [5] studied an elastic behavior of discs nonhomogeneous materials numerically via the Transfer Matrix Method (TMM). He presented analytical formulas in a compact form and including the different 
boundary conditions. Chen and Lin [6] investigated stress distributions analysis for spherical and cylinder pressure vessel made of FGMs with exponential function. Kurşun et al. [7] have analytically defined the thermal stress distribution of long hollow cylinders made of FGM under various loads and examined the effects of gradient parameters on stress distributions of FGM cylinders. Hassan and Keleş [8] modeled FGM solid cylinder subjected to external pressure by using dummy thermal loads in ANSYS. They obtained radial displacements with radial and tangential stresses from FEM and compared them with analytical results. Nejat et al. $[9,10]$ obtained closed-form solutions of radial and hoop stresses and radial deformation for spherical components made of FGMs for dissimilar values of material inhomogeneity constant. They compared the analytical results with the results of the FEM. Rahimi and Nejad [11], in their study, obtained the thermal stresses in the rotating cylinder made of FGM under inner and outer pressure. Jabbari et al. [12] came to a solution for thermal and mechanical loads in cylinder made of FGM using the Bessel function. Nie et al. [13] studied analytically to obtain the circumferential and the radial stresses of a cylinder or a sphere. They used rule of mixtures and Mori-Tanaka rule for material properties in FGMs. Ghannad and Gharooni [14] used based on third-order shear deformation theory to estimate the effects of strains and shear stresses for obtaining analytical results of axisymmetric cylinders for fixed boundary conditions. Afshar et al. [15] studied viscoelastic FG cylinder by using FEM to find the radial stress, radial deformation and tangential stress for various materials inhomogeneity constant. Najibi and Shojaeefard [16] in their study developed a new two dimensional-FGM material grading based on Mori-Tanaka rule and third-order transition function for a cylinder. They used FEM for stress analysis. Ansari and Kumar [17] investigated the bending analysis of functionally graded carbon nanotube (CNT) reinforced doubly curved singly ruled truncated rhombic cone. Anish et al. [18] investigated the failure mode prediction of sandwich plates subjected to uniform transverse load and bisinusoidal load using Tsai Wu's, Maximum stress, Hoffman's, Tsai Hill and Maximum strain failure criteria. Ansari and Kumar [19] studied the flexural analysis of functionally graded carbon nanotube (CNT)-reinforced doubly curved singly ruled composite truncated cone. Ansari et al. [20] presented a new mathematical model for doubly curved singly ruled functionally graded material moderately thick and deep cone. Demirbas and Apalak [21] investigated the thermo-elastic response based on the theory of elasticity in two-dimensional FGM circular plates. Demirbas and Apalak [22] carried out thermal residual stress analysis of functionally graded circular plates (FGCP) with temperature-dependent material properties. Apalak and Demirbas [23] investigated the thermal residual stress and deformations in functionally graded clamped circular plates.

In this study, hollow FGM cylinders exposed to internal and external pressure are discussed. The FGM cylinder properties are considered to vary with the simple power law function, unlike many other studies in the literature. The Poisson's ratio is considered to be constant. Radial displacements, radial and tangential (hoop) stresses were solved analytically as well as finite element method ANSYS APDL package program for numerically five different FGM types and the results were compared. The results between FEM and analytical solutions were well fit. In order to achieve optimum design with the results of the stress, equivalent stresses were calculated. Unlike many studies in the literature, an equation has been found for the inhomogeneity coefficient of the power function in this paper. And the effect of inhomogeneity constant on equivalent stresses was investigated. The results are presented in graphical form.

\section{THEORETICAL FORMULATION OF THE PROBLEM}

Think through a FGM cylinder element with inner and outer surfaces exposed to pressures $p_{a}$ and $p_{b}$ respectively, and its inner radius $a$, outer radius $b$, as shown in Figure 1. It is assumed that the cylinder has an axisymmetric plane strain condition. The problem will be formulated in polar coordinates $(r, \theta)$. And it was assumed that the material composition only changed according to the thickness. 


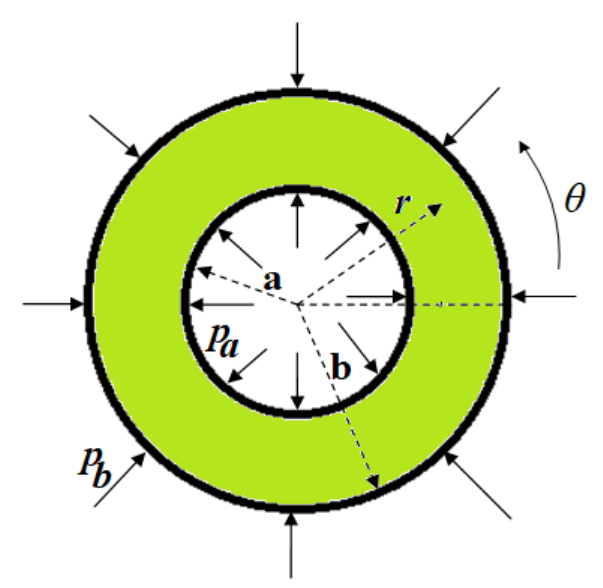

Figure 1. A section of FGM hollow cylinder exposed to pressures

In the radial direction $(r)$, while the elasticity modulus $(E(r))$ varies depending on a simple power function in Equation (1),

$E(r)=E_{a}\left(\frac{r}{a}\right)^{\beta}$ or $E(r)=a^{-\beta} E_{a} r^{\beta}$

$E_{A}=\frac{E_{a}}{a^{\beta}}, \quad E(r)=E_{A} r^{\beta}$

$\beta=\frac{\ln \left(\frac{E_{a}}{E_{b}}\right)}{\ln \left(\frac{a}{b}\right)}$.

$E_{a}$ and $E_{b}$ are the elasticity modulus of the materials in the inner and outer parts of the FGM cylinder respectively, while the $\beta$ is inhomogeneity constant which varies depending on the materials. The $\beta$ value will be zero when the cylinder material is homogeneous. Radial $\left(\varepsilon_{r}\right)$ and tangential $\left(\varepsilon_{\theta}\right)$ infinitesimal strains in polar coordinates $(r, \theta)$ for formulation

$\varepsilon_{r}=\frac{d u}{d r}=u_{r}^{\prime}$

$\varepsilon_{\theta}=\frac{u}{r}$

and equilibrium equation in polar coordinates is as in Equation (6)

$\frac{d \sigma_{r}}{d r}+\frac{\sigma_{r}-\sigma_{\theta}}{r}=0$

where $\sigma_{r}$ is the radial stress and $\sigma_{\theta}$ is the tangential stress. In the axisymmetric case, the stress-strain expressions are as follows for isotropic and radially functional graded material.

$\sigma_{r}(r)=C_{11} \varepsilon_{r}(r)+C_{12} \varepsilon_{\theta}(r)$

$\sigma_{\theta}(r)=C_{12} \varepsilon_{r}(r)+C_{11} \varepsilon_{\theta}(r)$.

Instead of $C_{11}$ and $C_{12}$ can be written the following equations for the cylinder

$C_{11}=\frac{E(r)(1-v)}{(1+v)(1-2 v)}$

$C_{12}=\frac{E(r) v}{(1+v)(1-2 v)}$. 
If Equations (7-10) are written in the equilibrium equation in Equation (6), the second order differential equation in Equation (11) is obtained

$\frac{(-1+\beta \lambda) u_{r}}{r^{2}}+\frac{(1+\beta) u_{r}^{\prime}}{r}+u_{r}^{\prime \prime}=0$

where $u_{r}$ is the radial displacement. The boundary conditions of the cylinder for this loading condition are

$\sigma_{r}(a)=-p_{a} ; \sigma_{r}(b)=-p_{b}$

After solving Equation (11) for boundary conditions in Equation (12), analytical expressions of $u_{r}, \sigma_{r}$ and $\sigma_{\theta}$ are obtained as follows;

$$
\begin{aligned}
& u_{r}(r)=\left\{\frac{2 \overline{2}^{\frac{1}{2}(-\beta+\xi+2)} p_{a} r^{\frac{1}{2}(-\beta-\xi)}\left(2 v^{2}+v-1\right)\left(b^{\xi}(-\beta+2 \lambda+\xi)+r^{\xi}(\beta-2 \lambda+\xi)\right)}{\left(a^{\xi}-b^{\xi}\right) E_{A}(v-1)\left((\beta-2 \lambda)^{2}-\xi^{2}\right)}\right\}+ \\
& \left\{\frac{2 b^{\frac{1}{2}(-\beta+\xi+2)} p_{b} r^{\frac{1}{2}(-\beta-\xi)}\left(2 v^{2}+v-1\right)\left(a^{\xi}(-\beta+2 \lambda+\xi)+r^{\xi}(\beta-2 \lambda+\xi)\right)}{\left(a^{\xi}-b^{\xi}\right) E_{A}(\nu-1)\left(\xi^{2}-(\beta-2 \lambda)^{2}\right)}\right\} \\
& \sigma_{r}(r)=\left\{\frac{a^{\frac{1}{2}(-\beta+\xi+2)} p_{a} r^{\frac{1}{2}(\beta-\xi-2)}\left(b^{\xi}-r^{\xi}\right)}{a^{\xi}-b^{\xi}}\right\}+\left\{\frac{\left.b^{\frac{1}{2}(-\beta+\xi+2)} p_{b^{\frac{1}{2}(\beta-\xi-2)}\left(a^{\xi}-r^{\xi}\right)}^{b^{\xi}-a^{\xi}}\right\}}{\left(a^{\xi}-b^{\xi}\right)\left(\xi^{2}-(\beta-2 \lambda)^{2}\right)}\right\} \\
& \sigma_{\theta}(r)=\left\{-\frac{a^{\frac{1}{2}(-\beta+\xi+2)} p_{a} r^{\frac{1}{2}(\beta-\xi-2)}\left((\beta-2 \lambda-\xi)(\lambda(\beta+\xi)-2) b^{\xi}+r^{\xi}(\beta-2 \lambda+\xi)(-\beta \lambda+\xi \lambda+2)\right)}{\left(a^{\xi}-b^{\xi}\right)\left(\xi^{2}-(\beta-2 \lambda)^{2}\right)}\right\}+ \\
& \left\{\frac{b^{\frac{1}{2}(-\beta+\xi+2)} p_{b} r^{\frac{1}{2}(\beta-\xi-2)}\left((\beta-2 \lambda-\xi)(\lambda(\beta+\xi)-2) a^{\xi}+r^{\xi}(\beta-2 \lambda+\xi)(-\beta \lambda+\xi \lambda+2)\right)}{2}\right\} \\
& \lambda=\sqrt{4+\beta^{2}-4 \beta \lambda} \\
& \lambda=\frac{v}{1-v} .
\end{aligned}
$$

Consider a cylinder with $a=40 \mathrm{~mm}$ for inner radius and $b=60 \mathrm{~mm}$ for outer radius, $p_{a}=0.5 \mathrm{GPa}$ internal pressure and $p_{b}=1 \mathrm{GPa}$ external pressure. The properties of materials used for the FGM cylinder are shown in Table 1. 
Table 1. Material properties of some metals and ceramics

\begin{tabular}{|l|l|l|l|}
\hline Metal & $\boldsymbol{E}(\boldsymbol{G P a})$ & $\boldsymbol{\rho}\left(\mathbf{k g} / \mathbf{m}^{3}\right)$ & $\boldsymbol{v}$ \\
\hline High Carbon Steel (HCS) & 207.5 & 7850 & 0.295 \\
\hline Nickel Alloy (NA) & 205 & 8890 & 0.310 \\
\hline Magnesium Alloy (MA) & 44.5 & 1845 & 0.281 \\
\hline Zinc Alloy (ZA) & 81.5 & 5975 & 0.3262 \\
\hline Copper Alloy (CA) & 130 & 8935 & 0.330 \\
\hline Ceramic & & \multicolumn{2}{|l}{} \\
\hline Magnesium Oxide (MgO) & 317 & 3580 & 0.17 \\
\hline Boron Carbide (B4C) & 460 & 2520 & 0.17 \\
\hline Zirkonium Diboride (ZrB $)$ & 340 & 6100 & 0.11 \\
\hline Silicon Carbide (SiC) & 480 & 3210 & 0.16 \\
\hline Aluminium Nitride (AlN) & 318 & 3260 & 0.25 \\
\hline
\end{tabular}

Five different types of FGM and their inhomogeneity constants $(\beta)$ are given in Table 2 . In this paper, the inner and outer surfaces is assumed to be metal and ceramic respectively for the material of hollow cylinder. Accordingly, it can be seen $\beta_{V}>\beta_{I V}>\beta_{I I I}>\beta_{I I}>\beta_{I}$ from Table 2 .

Table 2. Different $\beta$ coefficients for different metal-ceramic pairs

\begin{tabular}{|l|l|l|l|}
\hline & $\begin{array}{l}\text { Metal } \\
\text { (inner surface) }\end{array}$ & $\begin{array}{l}\text { Ceramic } \\
\text { (outer surface) }\end{array}$ & $\boldsymbol{\beta}$ \\
\hline FGM I & HCS & $\mathrm{MgO}$ & 1.045146489 \\
\hline FGM II & NA & $\mathrm{B}_{4} \mathrm{C}$ & 1.969362296 \\
\hline FGM III & CA & $\mathrm{AlN}$ & 2.206150207 \\
\hline FGM IV & ZA & $\mathrm{SiC}$ & 4.373207579 \\
\hline FGM V & MA & $\mathrm{ZrB}_{2}$ & 5.015120629 \\
\hline
\end{tabular}

Figure 2 shows a graph of the distribution of elasticity modulus along the thickness of the hollow cylinder for five different types of FGM according to the simple power rule. 


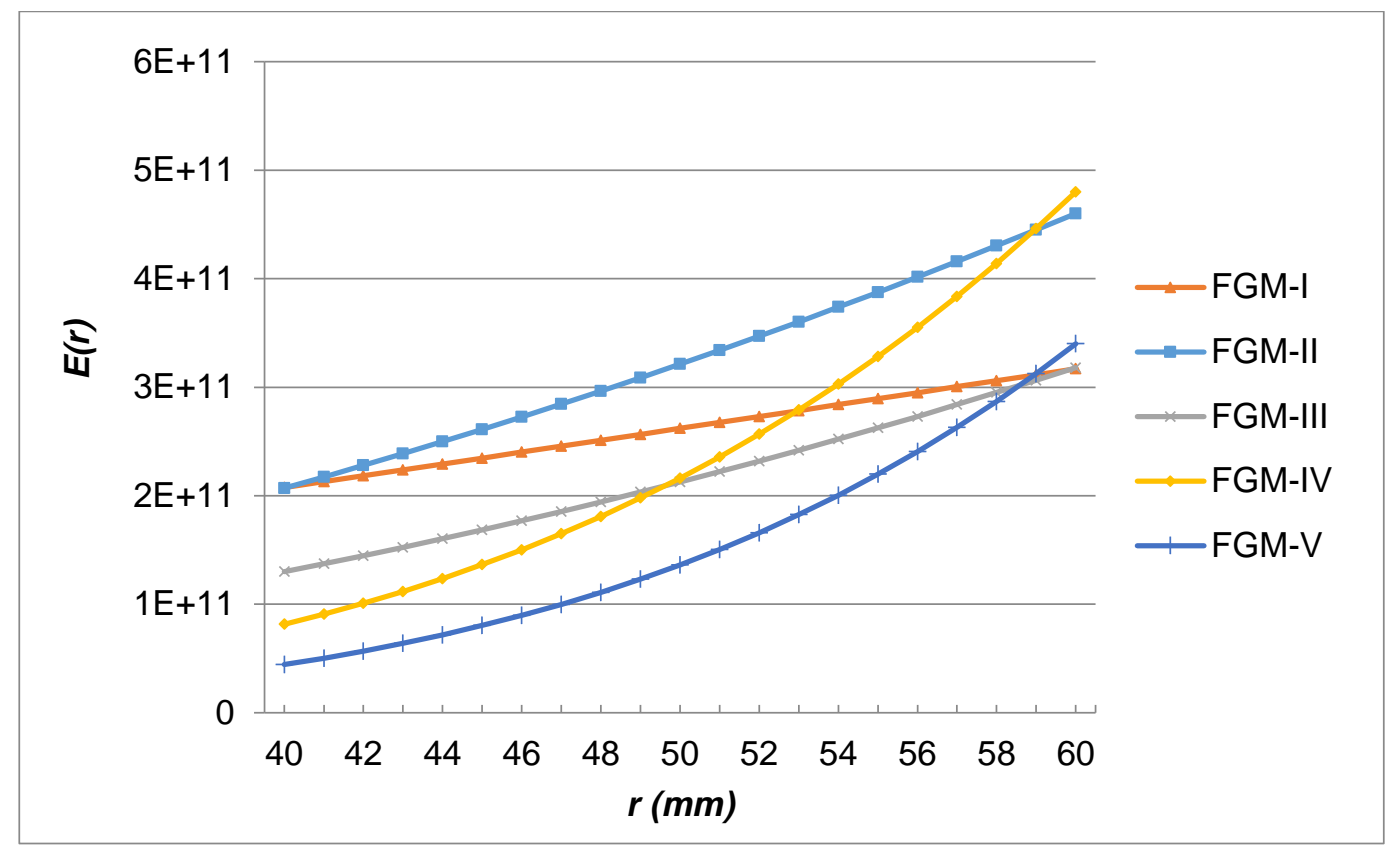

Figure 2. Distribution of elasticity modulus for different FGM models

The stress results found are useful for preventing failure in designing FGM hollow cylinders. The criteria for failure chosen are Von Misses failure criteria. The description of the criteria is that when the equivalent stress exceeds the yield stress, the material will fail. The equivalent stress is given by:

$\sigma_{e q}=\sqrt{\sigma_{r}^{2}+\sigma_{\theta}^{2}-\sigma_{r} \sigma_{\theta}}$.

\section{NUMERICAL ANALYSIS}

In this study, the ANSYS APDL package program, which is commonly used to solve engineering problems with the FEM. ANSYS allows the results to be quickly obtained on the computer before it goes into series production. Due to the symmetry for the finite element model, only one quarter of the cylinder geometry is modelled with $40 \mathrm{~mm}$ inner radius and $60 \mathrm{~mm}$ with outer radius. It is meshed with high density elements to obtain the stresses with high accuracy (Figure 3).

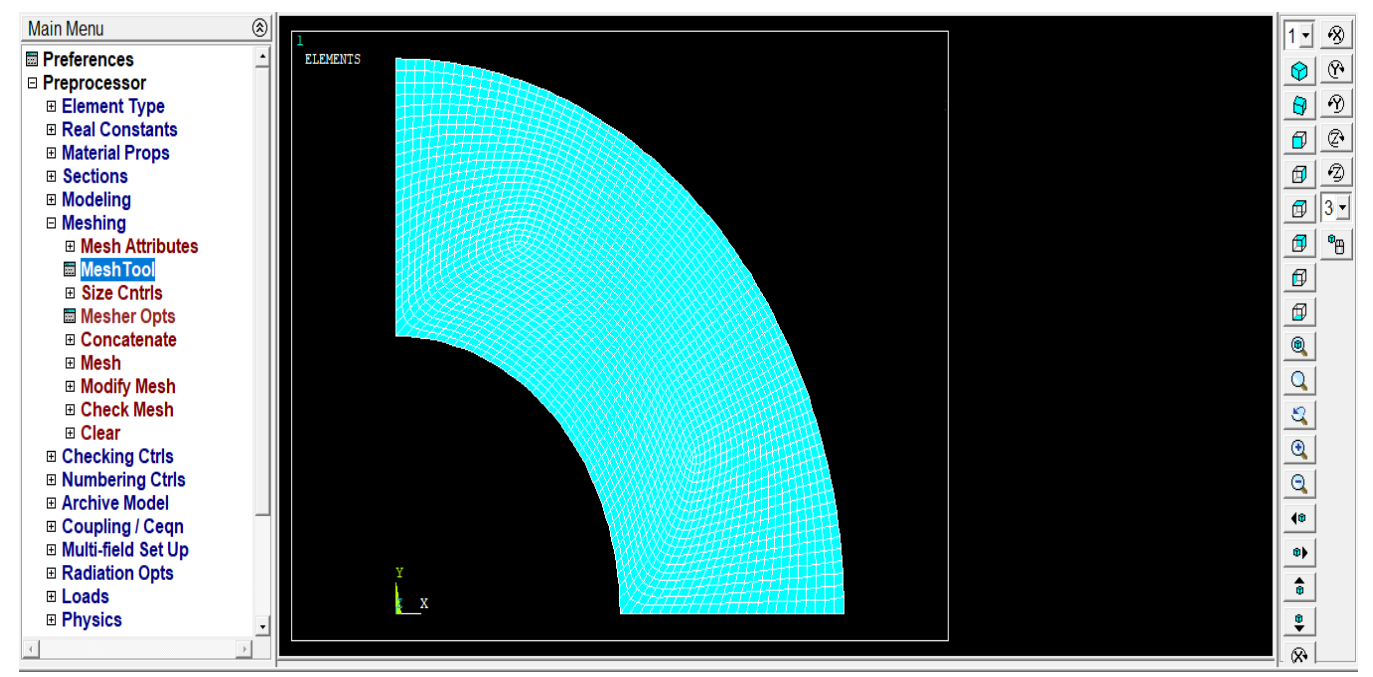

Figure 3. Creating part geometry and meshing

In the finite element cylinder model, other input data given are $p_{a}=0.5 \mathrm{GPa}$ internal pressure, $p_{b}=1 \mathrm{GPa}$ external pressure and $v=0.3$ Poisson's ratio. The elasticity modulus of the FGM cylinder is modelled in 
ANSYS APDL using MACRO because it changes in radial direction depending on the simple power rule function. The radial direction variation of the elasticity modulus is shown in Figure 4.

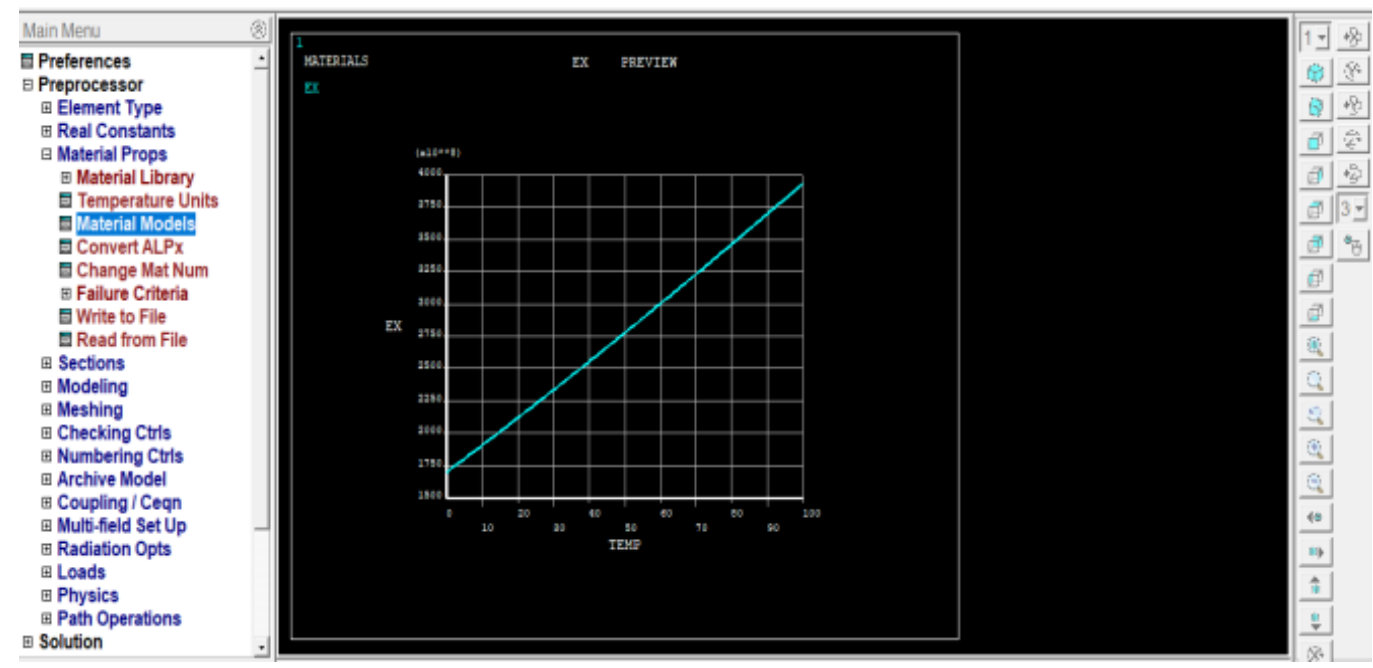

Figure 4. Graph of the variation of the elasticity modulus in the radial direction

\section{RESULTS AND DISCUSSION}

In this paper, analytical and FEM results were compared before the parametric study. Analytical and FEM radial and tangential stress results were similar for five different FGM types. It can be seen in Figure 5 radial stress values obtaining from ANSYS. Comparison of analytical and FEM radial stress values along the thickness of FGM I is shown in Figure 6. This shows that the analysis was carried out successfully.

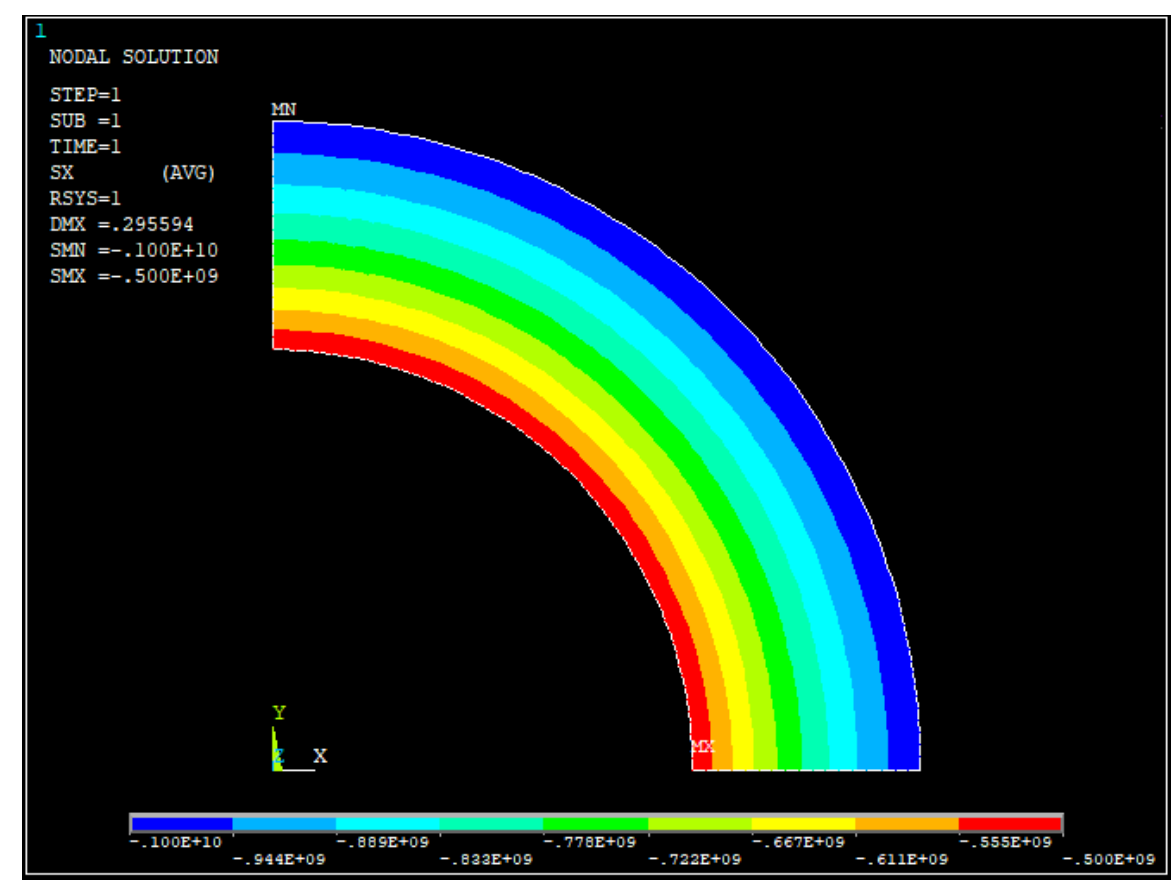

Figure 5. Radial stress values obtaining from ANSYS 


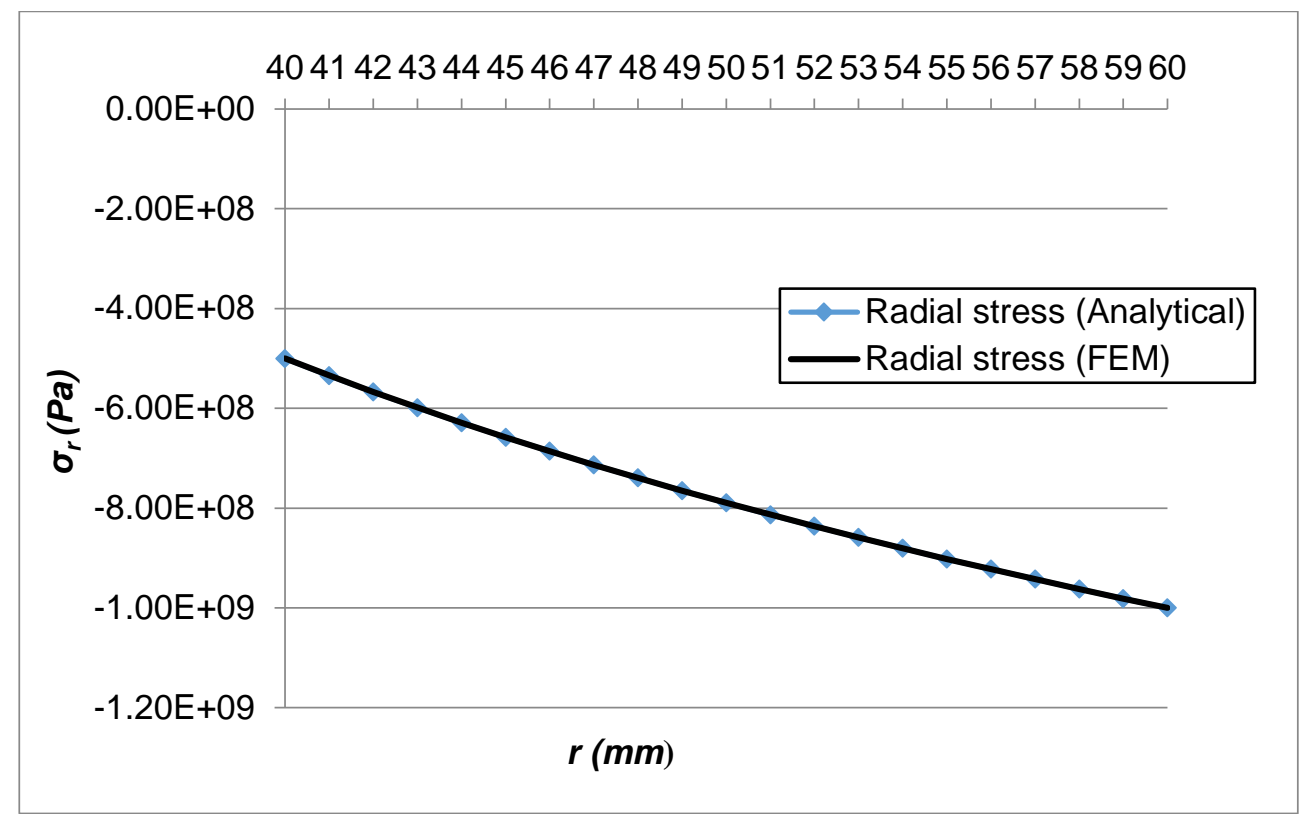

Figure 6. Comparison of analytical and FEM radial stress values along thickness for FGM I

After verification, for five different values $\beta$, radial stress along the radial direction can be seen in Figure 7. A hollow circular cylinder having uniform thickness $(20 \mathrm{~mm})$ is considered in this study. As expected, in consequence of such a pressure application the value of radial stress is $0.5 \mathrm{GPa}$ at internal side and 1 $G P a$ at external side. Radial stress values increases along the thickness of the hollow cylinder from internal side towards external side in Figure 7 for the reason that the elasticity modulus is an increasing function of the radius. In addition, we can observe that the radial stress values decreases as the inhomogeneity constants $(\beta)$ value increases. This is the result of being FGMs of the cylinder. Physically, this means that choosing the interior of the cylinder from high modulus elasticity increases the inhomogeneity coefficient and reduces the radial stresses. According to the results obtained in this paper, the material homogeneity constants have a significant effect on the mechanical behavior of the hollow cylinder made of FGM. The inhomogeneity coefficient should be increased for less radial stress value.

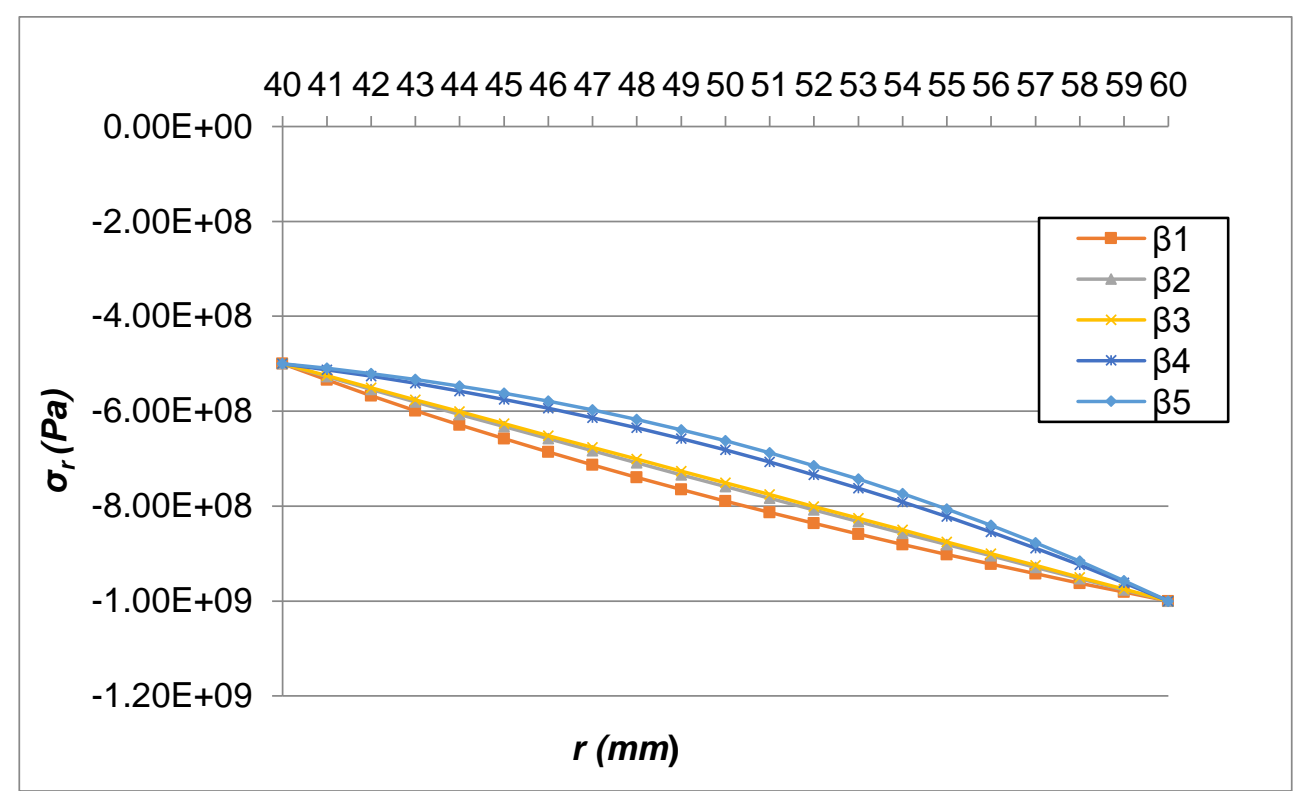

Figure 7. Variation of radial stress values along the thickness

Figures 8 and 9 show plots of the hoop stress and radial displacement along the radial direction for five different values of $\beta$, respectively. The hoop stress increases along the thickness of the hollow cylinder from internal side towards external side in Figure 8. We can say that the hoop stress value in the radial 
direction for FGM I changes very little and is almost constant. We can say that the hoop stresses for the hollow FGM cylinder has the same stress value at center of thickness and after the center point the hoop stresses decrease by decreasing the inhomogeneity constants $(\beta)$ at outer surface. We can say that the hoop stress values decrease with the increase of $\beta$ values before the center of thickness, and after the center of cylinder thickness the hoop stress values increase with the increase of $\beta$ values. Thus it can be concluded that hoop stresses are the same value in the center of the cylinder thickness for different $\beta$ values.

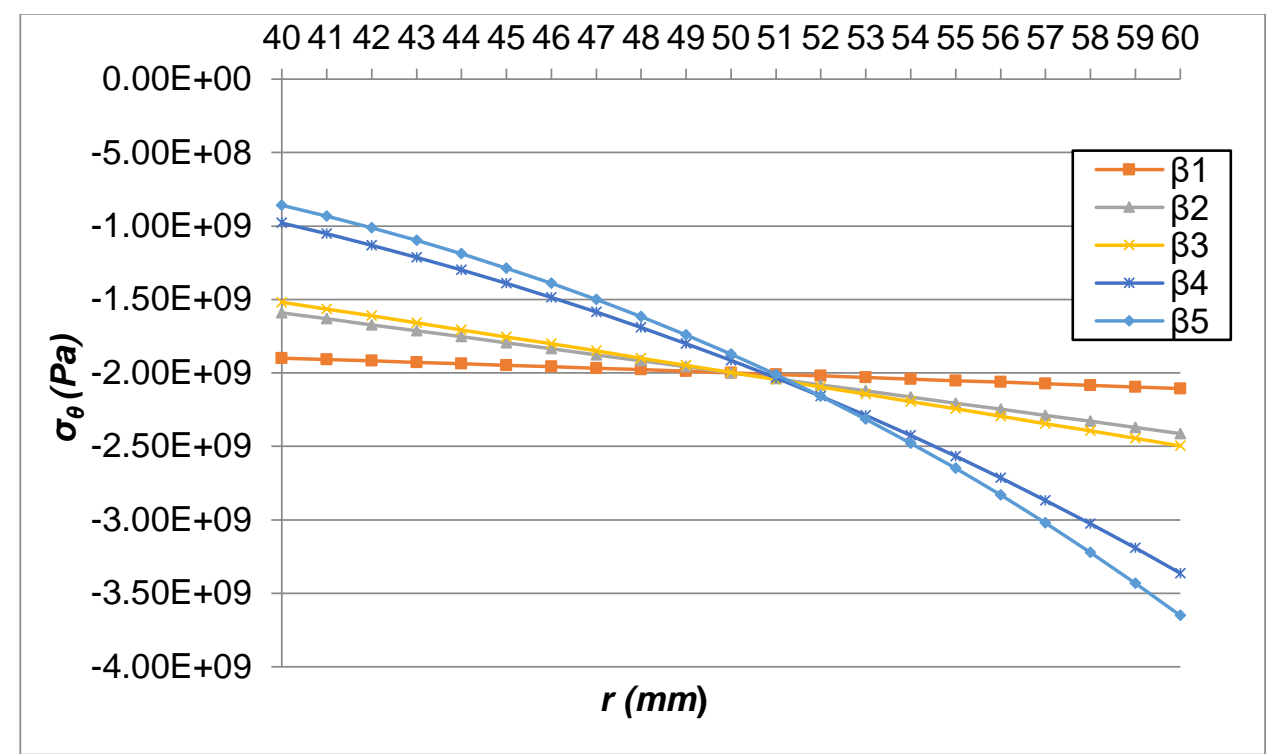

Figure 8. Distribution of hoop (tangential) stress along the thickness

Figure 9 explains the radial displacement along the thickness of hollow FGM cylinder for different $\beta$. We can note that the $u_{r}$ values decreases throughout the thickness of the FGM hollow cylinder from internal side towards external side. As shown in Figure 9 the highest radial displacement values occur at the highest $\beta$ value. Also, the radial displacements on the inner surface are larger than those on the outer surface because the inner surface is metal and the outer surface is ceramic.

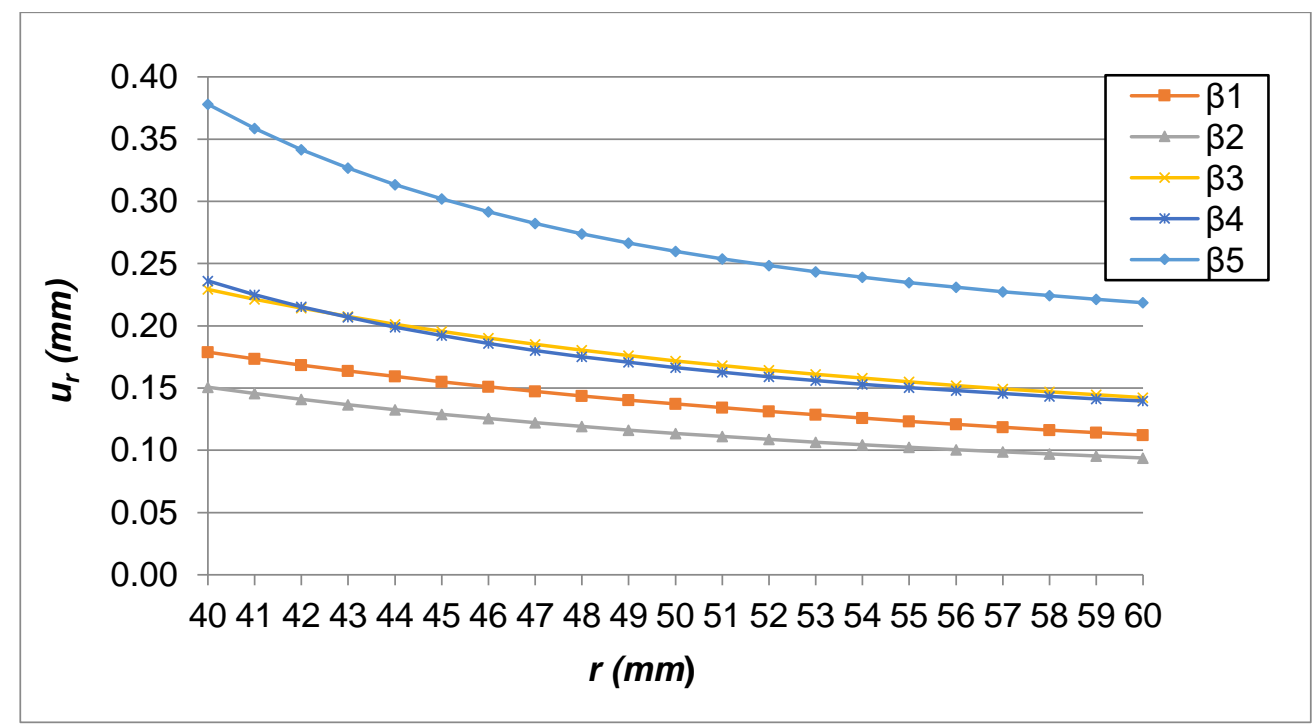

Figure 9. Distribution of radial displacement along the thickness 


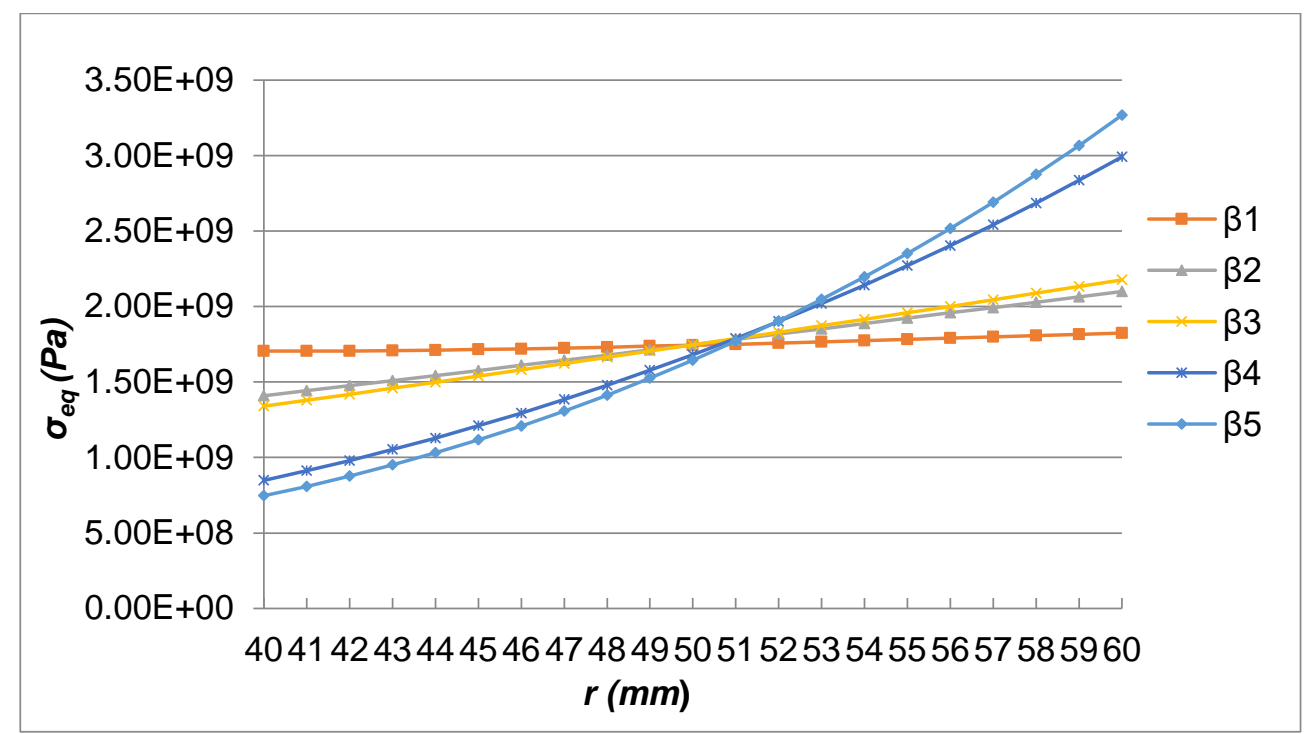

Figure 10. Distribution of equivalent stress along the thickness for different inhomogeneity constants

Figure 10 shows the equivalent stress values for different inhomogeneity constants along the thickness of FGM hollow cylinder. Figure 10 shows that the minimum equivalent stress values are occurred on the inner surface (metal surface). It can be observed that the equivalent stress values are increased as it moves towards the outer radius. This can be explained physically in the sense that the outer surface of the hollow cylinder is inclined to carry stress due to its high stiffness. Here again, it can be said that the equivalent stresses have the same value in the center of thickness. After the center point, the equivalent stresses increases with the increase of $\beta$, and the situation before the center point is the opposite. So the equivalent stresses decrease while the $\beta$ values increase.

\section{CONCLUSIONS}

In this paper, the elastic analysis of a hollow cylinder made of FGM, which is dependent on the simple power law function of the material properties exposed to the internal and external pressure, was made by assuming the plane strain. And the conclusions are as follows:

- For five different types of FGM with different material inhomogeneity coefficients $(\beta)$, closed form analytical expressions are provided which are very convenient for the easy determination of radial displacement, radial stress and tangential stress values.

- Also, in this study, numerical results were obtained with ANSYS, which is one of the finite element package programs. Analytical results were found to be appropriate with FEM results.

- By looking at the equivalent stress values, the type of FGM with the coefficient $\beta$ which is the most suitable for a hollow cylinder with such loading conditions can be determined.

- The obtained results, helps to prevent failure in the design of FGM hollow cylinder.

- The present results might be useful for mechanical engineers and material engineers in designing FGM hollow cylinders.

\section{CONFLICTS OF INTEREST}

No conflict of interest was declared by the author.

\section{REFERENCES}

[1] Timoshenko, S.P., Goodier, J.N., Theory of Elasticity, 3rd Ed., McGraw-Hill, New York, USA (1970).

[2] Tütüncü, N., Temel, B., "A Novel approach to stress analysis of pressurized FGM cylinders, disks and spheres", Composite Structures, 91:385-390, (2009). 
[3] Boğa, C., "Elastic analysis of a hollow cylinder made from functionally graded material exposed to internal pressure", ISVOS Journal, 2(1):56 - 66, (2018).

[4] Li, X.F., Peng, X.L., "A pressurized functionally graded hollow cylinder with arbitrarily varying material properties", Journal of Elasticity, 96:81-95, (2009).

[5] Boğa, C., "Analytical and numerical axisymmetric elastic stress analyses of stationary/rotating discs made of isotropic/orthotropic functionally graded materials by the transfer matrix method", $\mathrm{PhD}$. Thesis, Adana, Turkey, p177 (2016).

[6] Chen, Y.Z., Lin, X.Y., "Elastic analysis for thick cylinders and spherical pressure vessels made of functionally graded materials", Computational Materials Science, 44:581-587, (2008).

[7] Kurşun, A., Kara, E., Çetin, E., Aksoy, Ş., Kesimli, A., "Mechanical and thermal stresses in functionally graded cylinders", International Journal of Mechanical, Aerospace, Industrial, Mechatronic and Manufacturing Engineering, 8(2):303-308, (2014).

[8] Hassan, A., Keleş, İ., "FGM modelling using dummy thermal loads, applied with ANSYS APDL", Journal of Selcuk International Science and Technology, 1(1):10-18, (2017).

[9] Nejad, M.Z., Abedi, M., Lotfian, M.H., Ghannad, M., "An exact solution for stresses and displacements of pressurized FGM thick-walled spherical shells with exponential-varying properties", Journal of Mechanical Science and Technology, 26(12):4081-4087, (2012).

[10] Nejad, M.Z., Abedi, M., Lotfian, M.H., Ghannad, M., "Exact and numerical elastic analysis for the FGM thick-walled cylindrical pressure vessels with exponentially-varying properties", Archives of Metallurgy and Materials, 61(3):1649-1654, (2016).

[11] Rahimi, G.H., Nejad, M.Z., "Exact solutions for thermal stresses in a rotating thick-walled cylinder of functionally graded materials", Journal of Applied Sciences, 8(18):3267-3272, (2008).

[12] Jabbari, M., Bahtui, A., Eslami, M.R., “Axisymmetric mechanical and thermal stresses in thick short length FGM cylinders", International Journal of Pressure Vessels and Piping, 86:296-306, (2009).

[13] Nie, G. J., Zhong, Z., Batra, R.C., "Material tailoring for functionally graded hollow cylinders and spheres", Composites Science and Technology, 71:666-673, (2011).

[14] Ghannad, M., Gharooni, H., "Elastic analysis of pressurized thick FGM cylinders with exponential variation of material properties using TSDT", Latin American Journal of Solids and Structures, 12:1024-1041, (2015).

[15] Afshar, R., Bayat, M., Lalwani, R.K., Yau, Y.H., "Elastic behavior of glass-like functionally graded infinite hollow cylinder under hydrostatic loads using finite element method", Materials and Design, 32:781-787, (2011).

[16] Najibi, A., Shojaeefard, M.H., "Elastic mechanical stress analysis in a 2D-FGM thick finite length hollow cylinder with newly developed material model", Acta Mechanica Solida Sinica, 29(2):178191, (2016).

[17] Ansari, M.I., Kumar, A., "Bending analysis of functionally graded CNT reinforced doubly curved singly ruled truncated rhombic cone", Mechanics Based Design of Structures and Machines, 47(1):67-86, (2019).

[18] Anish, Kumar, A., Chakrabarti, A., "Failure mode analysis of laminated composite sandwich plate", Engineering Failure Analysis, 104:950-976, (2019). 
[19] Ansari, M.I., Kumar, A., "Flexural analysis of functionally graded CNT-Reinforced doubly curved singly ruled composite truncated cone", Journal of Aerospace Engineering, 32(2), (2019).

[20] Ansari M.I., Kumar, A., Chakrabarti, "Static analysis of doubly curved singly ruled truncated FGM cone", Composite Structures, 184:523-535, (2018).

[21] Demirbas M.D., Apalak M.K., "Investigation of the thermo-elastic response of adhesively bonded two-dimensional functionally graded circular plates based on theory of elasticity", Iranian Journal of Science and Technology, Transactions of Mechanical Engineering, 42(4):415-433, (2018).

[22] Demirbaş M.D., Apalak M.K., "Thermal stress analysis with finite difference method of functionally graded circular plates", International Journal of Mechanical And Production Engineering, 5(10):7884, (2017).

[23] Apalak M.K., Demirbaş M.D., "Thermal residual stresses in adhesively bonded in-plane functionally graded clamped circular hollow plates", Journal of Adhesion Science and Technology, 27(14):15901623, (2013). 